\title{
El experto en contenidos como instructor colaborador en el debriefing
}

\author{
The content expert as a collaborating instructor in debriefing \\ José M Maestre,* Elena Rojo RN,* Liébana Piedra,* Ignacio del Moral,* Robert Simon**
}

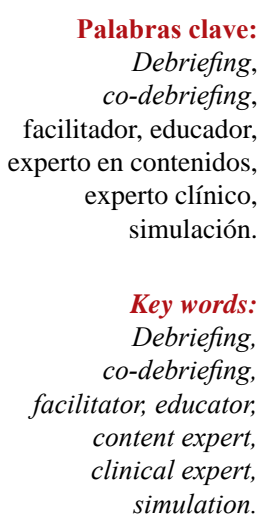

Declaración de

Conflicto de intereses:

Los autores no tienen

relaciones financieras con ninguna empresa comercial de productos o servicios relacionados con la simulación. El Hospital Virtual Valdecilla está afiliado con el Center for Medical Simulation; ambas son instituciones docentes sin ánimo de lucro que ofrecen programas de formación de instructores de simulación con matrícula a través del Institute for Medical Simulation.

\footnotetext{
* Hospital Virtual Valdecilla, Santander, España.

** Center for Medical Simulation, Boston, EUA.
}

Recibido: 23/02/2019 Aceptado: 15/03/2019

\section{RESUMEN}

Revisiones recientes de la literatura de simulación resaltan el papel crítico del informe (debriefing) en el proceso de aprendizaje. Por ello, ha aumentado el número de profesionales de la salud y otras disciplinas que se han capacitado como educadores para la realización del mismo. Sin embargo, a medida que aumenta el número y alcance de las actividades realizadas, no siempre es posible encontrar educadores que a la vez sean especialistas en el área específica de entrenamiento. Por ello, es común realizar los debriefings en colaboración con expertos en contenidos de diferentes áreas, que con frecuencia no tienen experiencia previa con educación basada en simulación. No se ha encontrado ninguna referencia específica a las oportunidades y retos propios de esta interacción. Por ello, el objetivo fue elaborar una guía para facilitar el debriefing entre educadores y expertos en contenidos que: 1) describa las ventajas de este tipo de colaboración, 2) discuta los desafíos comunes encontrados y 3) proponga herramientas prácticas para superar los desafíos específicos y, además, sugerir direcciones futuras para la investigación.

\section{ABSTRACT}

Recent simulation literature reviews highlight the critical role of debriefing in the learning process. As a consequence, there has been an increase in the number of health and allied professionals that have been trained to conduct debriefings. However, as the number and scope of the activities increase, it is not always possible to find expert debriefers who are also experts in the specific content area. Therefore, it is increasingly common for educators to collaborate with content experts in various fields in healthcare, who often have no previous experience with simulation-based education. No references to the particular opportunities and challenges of this interaction has been found. Therefore, the objective was to propose a guide to: 1) describe the advantages of this type of collaboration, 2) discuss common challenges and 3) overcome specific challenges; also, to suggest future directions for research.
"H oy es un día de celebración en nuestro centro de simulación, ya que por primera vez hemos realizado un curso de entrenamiento con el Departamento de Medicina Interna. Estoy especialmente feliz porque una renombrada experta clínica reservó un espacio en su agenda para participar y reflexionar sobre los pacientes. Además, los participantes se implicaron durante el caso y pronto, desde el comienzo del debriefing, se creó un ambiente de aprendizaje estimulante. El objetivo de aprendizaje era la toma de decisiones clínicas y todos nos involucramos en una conversación profunda tratando de comprender qué pasaba por sus mentes cuando se enfrentaron al paciente con dolor torácico y estaban intentando establecer el diagnóstico. Pero las cosas no se desarrollaron como esperaba. De repente, y sin previo aviso, nuestra invitada interrumpió la conversación y comenzó a compartir con nosotros las últimas pautas sobre el tratamiento de los pacientes con infarto. Había planeado con ella que yo conduciría la conversación y acordamos entender primero la perspectiva de los participantes para descubrir qué necesitaban aprender, así que intenté redirigir el enfoque. Desafortunadamente, no tuve éxito. La conversación se tornó en una conferencia magistral y continuó por un tiempo, y el debriefing se dirigió al protocolo de tratamiento en lugar de a lo que en realidad sucedió durante el caso".

\section{INTRODUCCIÓN}

El entrenamiento basado en simulación se está extendiendo en el pregrado, grado y postgra- 
do de muchas profesiones y disciplinas de la salud en todo el mundo. ${ }^{1}$ Ello es debido a que durante las dos últimas décadas, numerosas publicaciones han mostrado que promueve la integración de conocimientos y el desarrollo de habilidades procedimentales, además de mejorar el trabajo en equipo y la toma de decisiones clínicas, aumentando el grado de retención de lo aprendido cuando se compara con los métodos docentes tradicionales. ${ }^{2}$ Además, lo aprendido en el laboratorio de simulación se transfiere al entorno de trabajo, facilitando el cambio de la organización, mejorando los resultados en los pacientes y el retorno de la inversión. ${ }^{3}$ Esto ha provocado un aumento en la demanda de profesionales de enfermería, medicina, psicología, pedagogía u otras áreas de la salud formados como educadores para utilizar la simulación como herramienta de entrenamiento. ${ }^{1}$

Por otra parte, revisiones recientes de la literatura de simulación resaltan el papel fundamental del debriefing para lograr un aprendizaje efectivo; ${ }^{4,5}$ se entiende por debriefing la conversación entre dos o más personas que revisan un episodio real o simulado en la que los participantes analizan sus acciones y reflexionan sobre el papel de los procesos de pensamiento, habilidades psicomotrices y estados emocionales para mejorar o mantener su rendimiento en el futuro. ${ }^{6,7}$ Por ello y para dar respuesta a las necesidades planteadas, el número de profesionales que se han formado en debriefing ha aumentado incesantemente en los últimos años. ${ }^{8,9}$

Sin embargo, a medida que aumenta el número y alcance de las actividades realizadas, no siempre es posible encontrar profesionales de la salud capacitados en debriefing que a la vez sean expertos en los contenidos que se abordan durante la actividad de entrenamiento. Por lo tanto, es cada vez más común que estos profesionales realicen los debriefings en colaboración con expertos en contenidos de diferentes áreas de la salud, que con frecuencia no tienen experiencia previa con simulación. El impacto de esta colaboración en los participantes es determinante para el resultado del debriefing y para poder alcanzar los objetivos de aprendizaje planteados. ${ }^{10}$ En el caso descrito al inicio, la experta clínica se implicó en forma activa en la conversación con los participantes $y$, al tratar de compartir toda su valiosa experiencia, terminó dando una conferencia teórica. Los participantes se desconectaron lentamente del debriefing, ya que no pudieron participar de manera activa en la conversación, y el contenido se proporcionó antes de saber con exactitud cuál era la necesidad de conocimiento que los estudiantes tenían, si es que era así, lo que les impidió alcanzar el objetivo de diagnóstico y tratamiento correctos.

Por ello, se hace necesario entender las oportunidades y retos propios de esta colaboración, así como aplicar un enfoque estructurado que permita planificar de antemano el debriefing, mejorar la coordinación y asegurar que se consigan los objetivos docentes. Así, aunque alguna de las estrategias de mitigación descritas en la literatura para prevenir y enfrentar los retos propios del co-debriefing entre dos educadores se pueden aplicar a la interacción entre un experto en contenidos y un educador en simulación durante el debriefing, ${ }^{10}$ no hemos encontrado ninguna referencia a las oportunidades y retos particulares de esta interacción en las bases de datos MEDES (Medicina en Español), IBECS (Índice Bibliográfico Español en Ciencias de la Salud), DOCUMED (Documentación Médica Española), Scielo (Scientific Electronic Library Online) y MEDLINE (Base de Datos de la Biblioteca Nacional de Medicina Americana).

Por ello, el objetivo del presente artículo es proporcionar una guía con las consideraciones clave para conducir un debriefing eficaz realizado con la participación simultánea de un profesional de la salud o áreas relacionadas capacitado como educador en simulación (en adelante, "educador"), junto con un experto sobre la materia objeto de entrenamiento, sin formación específica como educador en simulación (en adelante, "experto en contenidos"). Nos hemos centrado en la dinámica de comportamiento entre ambos profesionales con el interés de: 1) describir las ventajas de este tipo de colaboración, 2) señalar los retos más frecuentes, 3) proporcionar herramientas prácticas para superar los retos específicos y, además, sugerir direcciones futuras para la investigación.

La base conceptual de este artículo es la literatura en el campo de la educación, psico- 
logía y sociología sobre la práctica reflexiva, en combinación con la experiencia de los autores en la aplicación del enfoque al debriefing "con buen juicio" y el desarrollo de educadores en simulación a nivel internacional. También se han fundamentado estas prácticas con los principios utilizados para desarrollar la herramienta de "Evaluación del Debriefing para Simulación en Salud" (EDSS) y los principios descritos para facilitar co-debriefing entre educadores. En los últimos dos años, los autores han capacitado más de 550 profesionales del área de la salud como educadores en simulación para utilizar este enfoque y trabajar de modo colaborativo con expertos en contenidos. La mayoría de los participantes han podido mostrar su competencia de manera confiable después de aproximadamente cuatro días de formación práctica, mientras que un nivel avanzado parece requerir mucho más tiempo.

\section{Beneficios de realizar un debriefing entre un educador y un experto en contenidos}

Una adecuada interacción entre un educador y un experto en contenidos durante el debriefing conduce a un aprendizaje profundo y efectivo en los participantes.

El educador contribuye de dos maneras fundamentales. ${ }^{11}$ Por un lado, creando un entorno de aprendizaje que promueva el compromiso auténtico por parte de los participantes para reflexionar de modo abierto sobre sus ideas y los motivos de su actuación. Cuando una persona permite que su rendimiento sea observado y analizado por otros está tomando riesgos psicológicos y para ello necesita percibir que su participación no tendrá implicaciones negativas. De este modo, es responsabilidad del educador establecer y mantener un ambiente de aprendizaje psicológicamente seguro. ${ }^{12}$ Por otro lado, explorando y analizando los procesos del pensamiento y estados emocionales que dieron lugar a las acciones observadas. Para lograrlo se necesita estructurar el debriefing de una manera organizada, provocar reflexiones estimulantes, brindar retroalimentación abierta y honesta sobre la actuación clínica y ayudar a los participantes a sostener o lograr un buen rendimiento en el futuro. ${ }^{13-15}$
El experto en contenidos puede contribuir de manera significativa a través de la discusión y/o enseñanza adaptada a las necesidades del participante una vez que se han comprendido los motivos que originaron la diferencia entre el rendimiento clínico esperado y el observado. Si los participantes no generan nuevas prácticas adecuadas a través de la discusión, entonces necesita proporcionar información específica sobre lo que contribuyó a la diferencia de rendimiento. Puede ser necesario dar una muy breve clase para compartir conocimientos, experiencia o datos de investigaciones que ilustren a los participantes cómo mejorar su rendimiento. El experto en contenidos aporta conocimiento y experiencia práctica en el tema que se aborda, que pueden incluir problemas clínicos, de comportamiento, trabajo en equipo, éticos, comunicación, profesionalismo o de otra naturaleza. ${ }^{16}$ Dada la gran amplitud de competencias a entrenar en el ámbito de la salud, a menudo es un reto encontrar una persona que reúna las características de ser educadora y experta en los contenidos específicos. Es por ello que, a menudo, la solución resulta en crear un equipo de dos personas que reúnan las habilidades requeridas por separado.

El éxito de esta interacción se sustenta, además, en unos valores o cualidades que caracterizan los intereses y sentimientos de ambos colaboradores, incluyendo respeto mutuo, confianza, interdependencia, empatía y vocación de servicio al participante como eje del encuentro de aprendizaje.

\section{Retos más frecuentes de realizar un debriefing entre un educador $y$ un experto en contenidos}

Si el educador y el experto en contenidos no están preparados para enfrentar los desafíos de la colaboración y la coordinación del debriefing, puede que los objetivos de aprendizaje no se logren o se produzca un efecto indeseado sobre los participantes. Algunos desafíos son comunes a la interacción entre dos educadores capacitados que realizan el debriefing de modo simultáneo, especialmente si utilizan metodologías diferentes. Sin embargo, dada la diferencia de formación base y enfoque docente que suele existir entre un educador en simulación y un 
experto en contenidos, los autores consideramos que existen algunas particularidades en la interacción derivadas de esta diferencia. Se describen a continuación las situaciones más prevalentes en nuestra experiencia.

Habitualmente, los expertos en contenidos que acceden a participar en las actividades basadas en simulación están muy comprometidos en ayudar a los participantes durante el debriefing. Así, es frecuente observar cómo contribuyen en forma activa a la conversación con muchos comentarios o preguntas. Sin embargo, a menudo tratan de abordar diferentes objetivos al mismo tiempo y la conversación salta de un punto a otro en lugar de progresar de manera lógica, lo que genera mucha confusión. Esto responde, a veces, a una falta de claridad entre los debriefers respecto a los objetivos clave de aprendizaje, a una falta de claridad de los roles durante la conversación o a aspectos relacionados con la jerarquía, normas sociales o la propia identidad profesional. Consideramos que es responsabilidad del educador aclarar de antemano cuáles son los objetivos, y estructurar y coordinar el flujo de la conversación.

Otra de las situaciones más frecuentes son las interrupciones de la conversación. A veces se interrumpe una secuencia de preguntas, lo que dificulta comprender a los participantes. Otras, se cambia el foco de la discusión, lo que resta profundidad al aprendizaje. Es común empezar la discusión antes de finalizar la fase de reacciones, por lo que los participantes pueden permanecer disgustados durante todo el debriefing. Generalmente, esto responde a agendas diferentes, normas de relación no explícitas, o como en el ejemplo anterior, falta de claridad de los roles, o aspectos relacionados con jerarquía o normas sociales.

Con frecuencia, la conversación se torna en una discusión sobre perspectivas teóricas y no sobre la experiencia vivida, tal y como ocurrió en el caso a estudio descrito al inicio. Ello impidió comprender los procesos de pensamiento de los participantes durante su desempeño y la enseñanza se volvió ineficaz, pues no se adaptó a sus verdaderas necesidades. Además, de este modo no se empleó todo la experiencia y conocimiento de la experta en medicina interna de la manera más eficiente. Esto suele ser debido a que, tradicionalmente, la capacitación en la mayoría de las profesiones en salud ha estado dominada por la transferencia de información de los profesores a los alumnos. ${ }^{17}$ Pero la investigación en varios campos muestra que las personas aprenden mejor como participantes activos responsables de su propio proceso de aprendizaje, en lugar de como receptores pasivos de conocimiento impartido por los maestros. ${ }^{18}$ El aprendizaje basado en simulación es, en esencia, experiencial, en el cual los participantes desarrollan su propia comprensión a través de la participación y la reflexión. ${ }^{6}$

En otras ocasiones, el debriefing sobre un objetivo dado finaliza sin una fase de síntesis y no se ayuda a relacionar el caso con formas para mejorar su práctica clínica futura. A menudo, esto se debe a una presión de tiempo o a agendas muy ambiciosas.

Por otra parte, y en ocasiones, se pierde consciencia de la situación. Los instructores se concentran de tal manera en mantener la estructura del debriefing (tratando de combinar la argumentación con la indagación cuando se quiere entender al participante utilizando un enfoque "con buen juicio", o mientras se da una minicharla teórica durante la conversación) que se pierde la consciencia de que algunas personas no participaban en la conversación, o que una o algunas de ellas parecían estar molestas por algo ocurrido durante el caso o la reflexión. Esto evita que los participantes se sientan estimulados a compartir sus pensamientos y preguntas sobre el rendimiento en la simulación. ${ }^{19}$

En algunas ocasiones, y queriendo contribuir al aprendizaje, se puede enfrentar el debriefing evaluando los conocimientos o prejuzgando la actuación del participante, formulando preguntas centradas en señalar los errores o que ocultan el propio punto de vista, en lugar de expresar curiosidad para entender la raíz de la actuación. ${ }^{13}$ En estas situaciones, los participantes suelen volverse reacios a compartir pensamientos y emociones por temor a ser avergonzados o humillados, y la conversación se torna ineficaz. ${ }^{20}$ Este patrón de comportamiento ocurre en cualquier profesional de la salud (los autores de este manuscrito incluidos) y a la vez puede ser más frecuente en el experto en contenidos dependiendo del número de oportunidades que se le han ofreci- 
do para reflexionar sobre sus propias actitudes y comportamientos para facilitar el aprendizaje de los adultos.

Por último, en ocasiones, alguno de los debriefers domina la conversación, bien porque se centra en un tema específico o en una parte de los participantes, en especial cuando el entrenamiento es interprofesional.

\section{Guía para la colaboración efectiva}

Cuando un experto en contenidos participa en una actividad de entrenamiento con simulación, con frecuencia, los educadores entusiastas intentan transmitirle toda su experiencia en unos pocos encuentros antes de que comience la actividad. Sin embargo, el proceso para adquirir las competencias necesarias para lograr debriefings efectivos requiere motivación, compromiso, apoyo y tiempo. ${ }^{21,22}$ Por ello, se considera que es necesario recalibrar el objetivo y las expectativas para este tipo de colaboración. ${ }^{23}$

Con base en los principios planteados en los apartados anteriores, en nuestros centros hemos incorporado una guía de orientación para integrar de manera efectiva a un experto en contenidos en el debriefing con un educador (Tabla 1). Se proponen unos comportamientos esenciales para lograr los objetivos de aprendizaje. Su aplicación en la práctica se fundamenta en dos ejes principales. Por un lado, en establecer la figura de coordinador del debriefing, y por otro, en enfatizar las estrategias preventivas frente a las reactivas cuando surgen diferencias de enfoque y la conversación no fluye como estaba planeado. Se propone que el rol de coordinador recaiga en el educador en simulación, por su formación específica, y que este realice un acuerdo de colaboración explícito con el experto en contenidos utilizando la guía como base.

Si bien es poco probable que una norma o reglamento cambie el comportamiento de las personas, ${ }^{24}$ esta guía pretende: 1) promover que el debriefing se planifique por anticipado, 2) ayudar a comprender el resultado deseado, 3) resaltar los desafíos comunes del debriefing conjunto, 4) establecer reglas de colaboración entre los instructores por adelantado, 5) identificar comportamientos que facilitan la realización de un debriefing conjunto efectivo, y 6) poner el foco en que los participantes reflexionen sobre su desempeño y los profesionales proporcionen retroalimentación adecuada a sus necesidades de aprendizaje. Con el marco de trabajo que se muestra, se propone, además, una forma práctica para abordar el debriefing y representa una oportunidad para reflexionar sobre su eficacia para promover el cambio en los adultos. ${ }^{14}$ Como el empleo de la simulación está en aumento para entrenar a equipos clínicos, residentes y estudiantes, y cada vez se requiere la participación de un mayor número de expertos en contenidos para abordar temas específicos, el objetivo a largo plazo es promover y ayudarles a ellos también a transformarse en "educadores reflexivos". Es decir, en individuos que aprenden a analizar sus propias suposiciones y rutinas mentales, que habitualmente se dan por asumidas, $y$ que son capaces de autocorregir y mejorar sus habilidades educativas. ${ }^{6}$

Por todo ello, se propone que el acuerdo formal sólo se realice una vez, siempre que ambas partes comprendan y compartan los principios para conducir la sesión, si bien para introducir de manera eficiente este marco de trabajo, se recomienda realizar una práctica repetitiva y reflexión sobre los comportamientos que ofrece la guía.

La guía comienza proponiendo una definición de trabajo sobre debriefing y los objetivos que se pretenden con su realización. Luego sugiere estrategias para promover y facilitar una colaboración efectiva siguiendo los principios utilizados para desarrollar la herramienta del EDSS. ${ }^{15}$

Los comportamientos dirigidos a "establecer y mantener un ambiente de aprendizaje participativo" son el elemento fundamental para mejorar la disposición de las personas a examinar y discutir sus pensamientos y acciones de manera crítica y abierta. ${ }^{7}$ Para crear un ambiente seguro desde el punto de vista psicológico, se invita al experto en contenidos a mantener un "principio básico" hacia sí mismo, el educador y los participantes de respeto mutuo. Se destaca el valorar la opinión experta de los instructores, mientras que al mismo tiempo se valora la perspectiva única de los participantes. Además, se pide mantener como confidencial toda la información relacionada 
Tabla 1. Guía para la facilitación del debriefing entre educadores y expertos en contenidos.

Concepto: Debriefing es una conversación entre dos o más personas para revisar un evento real o simulado, en la que los participantes analizan sus acciones y reflexionan sobre el papel de los procesos de pensamiento, las habilidades psicomotoras y los estados emocionales para mejorar o mantener el rendimiento en el futuro.

Objetivo: Esta guía tiene la intención de maximizar la calidad del debriefing, integrando de manera efectiva un educador experto en simulación con un experto en contenidos que no tiene certificación o antecedentes educativos basados en simulación, para promover un aprendizaje efectivo y la transferencia de las lecciones aprendidas a los sistemas sanitarios, con el fin de mejorar los resultados del paciente.

\section{Estrategias para promover y facilitar una colaboración efectiva durante el debriefing}

1. Establecer y mantener un ambiente de aprendizaje participativo.

a. Expresar el "principio básico" sobre los participantes: "creemos que todos los participantes son capaces, se preocupan por hacer bien su trabajo y quieren mejorar".

b. Utilizar los errores como misterios a resolver, no como delitos para ser castigados, evitando culpar a los participantes por sus acciones o usar un tono de voz crítico o acusatorio.

c. Mantener la "confidencialidad" con respecto al desempeño de los participantes durante el escenario y los debriefings.

2. Conducir el debriefing siguiendo una estructura y agenda comunes.

a. Establecer que el coordinador del debriefing sea el educador y situarse en un lugar donde ambos puedan establecer contacto visual entre ellos.

b. Discutir y mantener unos objetivos de aprendizaje compartidos.

c. Utilizar una metodología de debriefing compartida (comenzar por las reacciones, analizar lo sucedido y aplicar lo aprendido a prácticas futuras).

d. Evitar cambiar el objetivo de aprendizaje antes de finalizar el que se está discutiendo, interrumpir la cadena de pensamiento del otro instructor o hacer comentarios en más de dos ocasiones seguidas sobre un tema en particular (con raras excepciones).

3. Provocar discusiones estimulantes.

a. Planificar sobre temas de interés para la educación interprofesional y quién habla, con quién y cómo.

b. Presentar al experto en contenidos, incluidos sus antecedentes, el campo de especialización y el motivo por el que asiste.

c. Evitar el estilo tipo conferencia, dominar o hacerse cargo de la discusión, o hablar directamente o sólo con participantes de una profesión específica.

4. Identificar y explorar brechas de rendimiento.

a. Evitar centrarse sólo en corregir acciones y promover la discusión sobre los procesos de pensamiento de los participantes.

5. Ayudar a los participantes a lograr o mantener un buen rendimiento en el futuro.

a. Planificar cuándo el experto en contenidos contribuirá con su experiencia.

b. Evitar ignorar el rendimiento bueno o excelente, o pasar por alto puntos de aprendizaje clave.

6. Comportamientos a realizar si la colaboración no resulta según lo planeado (desacuerdos, interrupciones, cambios de tema, no participación).

a. Solicitar verbalmente permiso para interrumpir: “¿Podemos presionar el botón de pausa por un segundo?”.

b. Proporcionar una breve previsualización del tema sobre el que se desea hablar (por ejemplo: "Me gustaría hablar sobre el tema de la conversación").

c. Indicar lo que se observa (por ejemplo: “Observo que estamos cambiando el tema de la discusión”).

d. Compartir el propio punto de vista (por ejemplo: "Creo que es importante hablar sobre estrategias de comunicación antes de continuar con las dosis").

e. Preguntar y escuchar el punto de vista del otro instructor: "Me pregunto qué piensas". 
con personas específicas sobre su rendimiento clínico o comentarios durante el debriefing y aclarar el uso de las grabaciones. ${ }^{25}$

$\mathrm{Si}$ bien un estilo particularmente efectivo para entender la perspectiva de los participantes sobre lo ocurrido para "identificar y explorar brechas de rendimiento" es unir la argumentación con la indagación, ${ }^{14}$ no se considera útil dentro de los primeros pasos enseñar este estilo al experto en contenidos. En esta etapa inicial, se estima de suficiente valor para explorar el respetar al profesional y expresar curiosidad por entender los motivos de su actuación.

Un comportamiento clave para "ayudar a los participantes a lograr o mantener un buen rendimiento en el futuro" consiste en que el educador, una vez que ha identificado los motivos que condujeron al participante a realizar las acciones observadas durante la simulación, señale de modo claro y directo al experto en contenidos que "lleve el peso" de la conversación, para que contribuya con su experiencia discutiendo $y / o$ enseñando conocimientos $y$ habilidades adaptadas a las necesidades específicas del participante. Si este muestra una laguna de conocimiento en un área específica como causa de su actuación, el educador debe señalar que puede ser el momento para dar una miniconferencia sobre el tema. ${ }^{15,16}$

Resulta extremadamente útil discutir con antelación la forma de reaccionar si la conversación no va según lo planeado. ${ }^{10}$ Pausar el debriefing, nombrar la dinámica observada y promover una conversación transparente delante de los participantes no sólo resulta muy útil para reestructurar el debriefing, sino que además modela una forma de abordar diferencias de opinión o situaciones difíciles.

Otra estrategia complementaria con las anteriores consiste en pactar un tiempo tras finalizar la actividad para reflexionar sobre la propia interacción y realizar un debriefing del debriefing. Los métodos para este proceso se pueden consultar en la literatura ${ }^{26}$ y no están considerados como parte de esta guía de actuación.

\section{Investigaciones futuras}

En este artículo se han resaltado los principales desafíos de la interacción entre expertos docen- tes y de contenidos durante el debriefing, y las posibles estrategias preventivas y reactivas, pero aún quedan muchas preguntas por resolver. Resulta necesario identificar las prácticas que se asocian a un mayor número de comportamientos reflexivos de los participantes o las que producen mejores resultados de aprendizaje. También, identificar estrategias para abordar retos particulares como diferencias jerárquicas, culturales o relacionadas con el contexto de la organización. Si bien se anima a la comunidad de simulación para abordar estas y otras preguntas de investigación, proponemos que se utilice la guía para promover debriefings más coordinados y eficaces.

\section{CONCLUSIÓN}

En resumen, es cada vez más común encontrar expertos en contenidos específicos que participan durante actividades de entrenamiento basadas en simulación en colaboración con educadores de simulación. Entendiendo que los enfoques metodológicos varían según los centros y que aún se necesitan más investigaciones para determinar las prácticas específicas que logran un aprendizaje más efectivo, la guía de orientación que aquí se presenta pretende ayudar a crear conciencia sobre algunos principios fundamentales de dicha colaboración y promover el desarrollo de hábitos reflexivos entre los profesionales con vocación docente.

\section{REFERENCIAS}

1. Del Moral I, Maestre JM. A view at the practical application of simulation in professional education. Trends Anaesth Crit Care. 2013; 3: 146-151.

2. Sancho R, Rábago JL, Maestre JM, Del Moral I, Carceller JM. Integración de la simulación clínica en el programa formativo de la especialidad de Anestesiología y Reanimación. Rev Esp Anestesiol Reanim. 2010; 57: 656-663.

3. McGaghie WC, Draycott TJ, Dunn WF, Lopez CM, Stefanidis D. Evaluating the impact of simulation on translational patient outcomes. Simul Healthc. 2011; 6: S42-47.

4. Cook DA, Hamstra SJ, Brydges R, Zendejas B, Szostek $\mathrm{JH}$, Wang AT, et al. Comparative effectiveness of instructional design features in simulation-based education: systematic review and meta-analysis. Med Teach. 2013; 35: e867-898.

5. Issenberg BS, Scalese RJ. Best evidence on high-fidelity simulation: what clinical teachers need to know. Clin Teach. 2007; 4: 73-77. 
6. Maestre JM, Szyld D, Del Moral I, Ortiz G, Rudolph JW. La formación de expertos clínicos: la práctica reflexiva. Rev Clin Esp. 2014; 214: 216-220.

7. Rudolph JW, Raemer DB, Simon R. Establishing a safe container for learning in simulation: the role of the presimulation briefing. Simul Health. 2014; 9: 339-349.

8. Opazo ME, Rojo E, Maestre JM. Modalidades de formación de instructores en simulación clínica: el papel de una estancia o pasantía. Educ Med. 2017; 18: 22-29.

9. Mukunda S, Shustak R, Szyld D, Del Moral I, Maestre J. Reflexiones sobre una rotación educativa en simulación médica. FEM. 2015; 18: 169-171.

10. Cheng A, Palaganas J, Eppich W, Rudolph J, Robinson T, Grant V. Co-debriefing for simulation-based education: a primer for facilitators. Simul Healthc. 2015; 10: 69-75.

11. Rudolph JW, Simon R, Raemer DB, Eppich WJ. Debriefing as formative assessment: closing performance gaps in medical education. Acad Emerg Med. 2008; 15: 1010-1016.

12. Rudolph JW, Foldy EG, Robinson T, Kendall T, Taylor SS, Simon R. Helping without harming: the instructor's feedback dilemma in debriefing: a case study. Simul Health. 2013; 8: 304-316.

13. Rudolph JW, Simon R, Rivard P, Dufresne RL, Raemer DB. Debriefing with good judgment: combining rigorous feedback with genuine inquiry. Anesthesiol Clin. 2007; 25: 361-376.

14. Maestre JM, Rudolph JW. Theories and styles of debriefing: the good judgment method as a tool for formative assessment in healthcare. Rev Esp Cardiol (Engl Ed). 2015; 68: 282-285.

15. Brett-Fleegler M, Rudolph J, Eppich W, Monuteaux M, Fleegler E, Cheng A, et al. Debriefing assessment for simulation in healthcare: development and psychometric properties. Simul Healthc. 2012; 7: 288-294.

16. Rudolph JW, Simon R, Dufresne RL, Raemer DB. There's no such thing as "nonjudgmental" debriefing: a theory and method for debriefing with good judgment. Simul Healthc. 2006; 1: 49-55.

17. McGaghie WC, Issenberg SB, Cohen ER, Barsuk JH, Wayne DB. Does simulation-based medical education with deliberate practice yield better results than traditional clinical education? A meta-analytic comparative review of the evidence. Acad Med. 2011; 86: 706-711.
18. Motola I, Devine LA, Chung HS, Sullivan JE, Issenberg SB. Simulation in healthcare education: a best evidence practical guide. AMEE Guide No. 82. Med Teach. 2013; 35: e1511-1530.

19. Grant VJ, Robinson T, Catena H, Eppich W, Cheng A. Difficult debriefing situations: a toolbox for simulation educators. Med Teach. 2018; 40: 703-712.

20. Roussin CJ, Larraz E, Jamieson K, Maestre JM. Psychological safety, self-efficacy, and speaking up in interprofessional health care simulation. Clin Simul Nurs. 2018; 17: 38-46.

21. Eppich WJ, Hunt EA, Duval-Arnould JM, Siddall VJ, Cheng A. Structuring feedback and debriefing to achieve mastery learning goals. Acad Med. 2015; 90: 1501-1508.

22. Natal B, Szyld D, Pasichow S, Bismilla Z, Pirie J, Cheng A. Simulation fellowship programs: an international survey of program directors. Acad Med. 2017; 92: 1204-1211.

23. Zigmont J, Oocumma N, Szyld D, Maestre JM. Educator training and simulation methodology courses. In: Palaganas JC, Maxworthy JC, Epps CA, Mancini ME, editors. Defining excellence in simulation programs. Philadelphia: Wolters Kluwer Lippincott Williams \& Wilkins; 2014. pp. 548-559.

24. Rojo E, Maestre JM, Díaz-Mendi AR, Ansorena L, Del Moral I. Innovando en procesos asistenciales y seguridad del paciente mediante simulación clínica. Rev Calid Asist. 2016; 31: 267-278.

25. León-Castelao E, Maestre JM. Prebriefing en simulación clínica: análisis del concepto y terminología en castellano. Educ Med. 2019. Avance online.

26. Cheng A, Grant V, Huffman J, Burgess G, Szyld D, Robinson $\mathrm{T}$, et al. Coaching the Debriefer. Simul Healthc. 2017; 12: 319-325.

Correspondencia:

José M. Maestre

Hospital Virtual Valdecilla

Av. de Valdecilla $\mathrm{s} / \mathrm{n}$,

39008, Santander, España.

E-mail: jmmaestre@hvvaldecilla.es 